ANL-5901

Instruments

AEC Research and

Development Report

ARGONNE NATIONAL LABORATORY

P. O. Box 299

Lemont, Illinois

INSTALLATION AND INTERFERENCE PROBLEMS

IN REACTOR INSTRUMENTATION SYSTEMS

by

William K. Brookshier

August, 1958

Operated by The University of Chicago

under

Contract $W-31-109-e n g-38$ 


\section{DISCLAIMER}

This report was prepared as an account of work sponsored by an agency of the United States Government. Neither the United States Government nor any agency Thereof, nor any of their employees, makes any warranty, express or implied, or assumes any legal liability or responsibility for the accuracy, completeness, or usefulness of any information, apparatus, product, or process disclosed, or represents that its use would not infringe privately owned rights. Reference herein to any specific commercial product, process, or service by trade name, trademark, manufacturer, or otherwise does not necessarily constitute or imply its endorsement, recommendation, or favoring by the United States Government or any agency thereof. The views and opinions of authors expressed herein do not necessarily state or reflect those of the United States Government or any agency thereof. 


\section{DISCLAIMER}

Portions of this document may be illegible in electronic image products. Images are produced from the best available original document. 


\title{
INSTALLATION AND INTERFERENCE PROBLEMS \\ IN REACTOR INSTRUMENTATION SYSTEMS
}

by

William $K$. Brookshier

\begin{abstract}
Reactor control instrumentation systems may be subject to a variety of extraneous signals. Five types are discussed: (1) signals involving high-frequency components which may gain access to critical circuits through wiring that lacks suitable RF filtering; (2) current signals due to mechanical shocking or flexing of cables: (3) extraneous ion chamber signals, including displacement currents due to fluctuations in the polarizing voltage, as well as currents caused by mechanically induced changes in ion chamber capacity; (4) signals whose origin is magnetic induction into cable loops; and (5) signals involving magnetic induction and ohmic induction into auxiliary loops created by multiple ground connections.
\end{abstract}

The emphasis is placed on magnetic induction and means of eliminating it.

\section{INTRODUCTION}

People who work with radiation detection systems a round nuclear reactors and other sources of radiation have probably been plagued at one time or another with the pickup of spurious electromagnetic signals. It is not uncommon for AC power devices, such as brush-type motors, power relays, circuit breakers, or anything that may involve the generation of sparks, to produce extraneous counts in a pulse-counting system. It is equally possible for such sources of electromagnetic fields to interfere with the proper operation of the DC control instrumentation of a reactor.

A linear DC-current-measuring channel, consisting of an ionization chamber feeding a DC-current signal to an electrometer circuit may, particularly when the current level is $10^{-9}$ amp or below, exhibit sudden jumps when certain $A C$ power devices in the vicinity are operated. If the channel is used only for monitoring and recording the power level of the reactor, the fluctuations on the recorder chart may not be of great concern, except insofar as they may be misinterpreted as changes in power level. If the channel is a safety channel with trip circuits for shutting down the reactor at excessive power levels, the interfering signals may result in undesired reactor shutdowns. 
The process of locating and correcting difficulties of this type usually involves a number of man-hours in excess of that required for the proper system layout originally, and serves to accent the need for a somewhat better understanding of the nature of the problems associated with such systems.

The instrument which is usually the most readily affected by such spurious electromagnetic signals, and other sources of disturbances, is the $\log \mathrm{N}$ and period channel. This usually consists of an ionization chamber feeding a DC-current signal to a device which gives an output voltage proportional to the logarithm of the input current, after which the derivative is taken to obtain a signal proportional to the reciprocal period of an exponential input. Three factors contribute to this instrument's sensitivity to spurious signals. First, it usually must operate part time in a low-current region where impedance levels are high. Second, it has extremely high sensitivity because of the derivative function. Third, it involves a nonlinear device, the logarithm function, which can produce rectification of high-frequency signals that might otherwise pass unnoticed.

Although experience has seemed to indicate that magnetic induction into cable loops is one of the most prevalent types of spurious signal pickup, there are several other effects that occur frequently. These involve conduction of high-frequency transients into sensitive circuits that lack RF filtering, cable effects, and extraneous ion chamber signals. These will be discussed before tackling the more difficult problem of magnetic induction.

\section{RF FILTERING}

It is possible for spurious signals, which may involve frequencies in the megacycle region, to gain admittance to the critical parts of a circuit through filament and power supply leads, or other wiring. Fig. I is a block diagram of a period meter typical of the types used on most of Argonne's reactors. The DC-current signal from a neutron-sensitive chamber feeds directly into a conventional feedback circuit containing a logarithmic diode in a feedback connection. The incremental input resistance, or ratio of a small change of applied voltage to the resulting small change of current, is given by $R_{i}=\frac{K_{1}}{2.3 I}$, where $K_{1}$ is the volts per decade diode constant, and I is the DC current through the diode. This follows from taking the derivative of $V$ with respect to $I$ in the current-voltage relation $V=K_{1} \log _{10} I+K_{2}$. If we substitute numbers of $0.8 \mathrm{v}$ and $10^{-11} \mathrm{amp}$ for $K_{1}$ and $I$, respectively, the value for $R_{i}$ becomes $0.35 \times 10^{11}$ ohms. The value of $0.8 \mathrm{v}$ for $K_{1}$ results from the use of 10 silicon diodes in series as the $\log$ element, while $10^{-11}$ amp is the low end of the current range covered. The input resistance is $R_{i}$ divided by $1+A_{1}$ for low frequencies. For high frequencies, the feedback connection does not reduce the input impedance, 
and any electromagnetic field within the preamp enclosure of such a high frequency will very easily impress a voltage across the input, which will then be partially rectified by the nonlinearity of the log diode. The logical cure is to keep the interior of the preamplifier unit as field-free as possible over a wide frequency range. For this reason, all of Argonne's period meter preamps have used RF filters - simple $L$ and $C$ of about $100 \mu \mathrm{h}$ and $0.1 \mu f$ - in most of the leads into the preamp. The result of this filtering has been that all of the period meters, when bench tested without any signal cables connected, have been entirely insensitive to disturbances which affected earlier instruments.

\section{CABLES}

This pleasant situation may no longer exist when cables are connected. The system almost always becomes vulnerable in various degrees to certain disturbances. One such disturbance is mechanical shocking or flexing of the cables. This is probably due to charge transfer resulting from the relative motion between the dielectric and shield braid of the current signal cable, and not due to changes in capacity. Momentary currents as high as $10^{-11}$ amp may be generated by extreme flexing of ordinary coaxial cables. The commercially available "low noise" cables, which contain a graphite coating between the dielectric and the shield braid, reduce the magnitude of this effect by at least a factor of 10 , and should be used for the critical current signal cables. The graphite presents a slight hazard, since it can become smeared into a leakage path across otherwise good insulation in the process of placing a connector on the cable, but care and cleanliness will usually avoid this trouble.

In extremely critical applications where even the "low noise" cables may be troublesome in this manner, the only recourse may be to install the cables where they will not be subject to mechanical shock. If this cannot be done, the use of rigid coaxial cables may be warranted.

Most of the coaxial cables of better quality are made with a tight shield braid, or even a double braid, which gives practically complete protection against the penetration of electric fields. Some, however, may use a loose weave that leaves gaps and results in very incomplete shielding. Cables such as these will almost invariably cause trouble in critical systems involving high impedances.

\section{EXTRANEOUS ION CHAMBER SIGNALS}

In its simplest form, an ion chamber consists of two plates separated by a gas-filled space in which the collection of ions takes place. The electric field set up in the space for the purpose of collecting the ions also 
guarantees the existence of physical capacity, which represents the chamber. For a chamber of applied voltage $V$ and capacity $C$, the charge is given by $Q=C V$. In the general case,

$$
I=\frac{d Q}{d t}=C \frac{d V}{d t}+V \frac{d C}{d t}
$$

Both of these components of current, one depending upon rate of change of applied voltage, and the other upon rate of change of capacity, represent undesired components, since the desired signal is the directly collected charge.

Minimizing the first component, $\mathrm{C} d V / d t$, may place rather severe requirements upon the stability of the voltage source used to polarize the ion chamber. For a chamber of $100-\mu \mu$ capacity, a voltage rate of only 0.01 volt per second will generate an error current of $10^{-12}$ amp. This could be serious in a linear measuring channel and is even more serious in a period measuring channel. Even in the case of an electronically regulated supply, the output of which is known to be stable to a few millivolts for a wide range of line-voltage variation, there is no guarantee that highfrequency transients will not be present.

Some people, involved with the touchiness of pexiod circuits, have settled the problem of ion chamber voltage stability, along with many other pickup problems, by inserting long slowing-down time constants in the period circuits. This naturally results in a longer elapsed time between the receiving of a dangerous period signal, and the action of shutting down the reactor.

The philosophy at Argonne has been to keep the response time of the period-measuring device only as long as required by the statistics in the ion chamber current. Incremental time constants in the order of 0.2 to $0.4 \mathrm{sec}$ have been maintained ove $x$ the current range of $10^{-3}$ down to $10^{-10} \mathrm{amp}$, with an increase by a factor of two at $10^{-11} \mathrm{amp}$. With a period circuit as fast as this the stability of the ion chamber voltage is particularly critical.

In some systems a step change of only 1 millivolt, out of a value of several hundred volts, is all that is required to produce an error period signal equal to full-scale deflection when operating at the low end of the current range. This degree of stability has been obtained in reasonably simple electronic regulators. However, where possible, the use of an additional $R C$ integrating time constant in series with the output of the voltage supply to the chamber is very desirable, and may relax the stability requirements of the regulated supply by a very large factor. By the use of such a time constant $R C$, the maximum value of the error current fed as displacement current through the chamber capacity $\mathrm{C}_{\mathrm{C}}$ is

$$
\mathrm{I}=\frac{\mathrm{C}_{\mathrm{C}}}{\mathrm{RC}} \Delta \mathrm{V}_{\mathrm{S}} \text {, }
$$

where $\Delta V_{S}$ represents a step change in the voltage supply. 
Minimizing the second term, $\mathrm{V} d \mathrm{C} / \mathrm{dt}$, in equation (1), may place severe requirements upon the mechanical stability of the ion chamber. If an ion chamber with constant voltage $V_{c}$ undergoes a step change in capacity $\Delta C_{C}$, a charge $\Delta Q=V_{C} \Delta C_{C}$ is transferred to the chamber. If this charge flows through a circuit of input capacity $C_{i}$, it will produce a step change of voltage:

$$
v_{i}=v_{c} \frac{\Delta C_{c}}{C_{i}}
$$

The value of capacity change $\Delta C_{c}$ required to produce a voltage step of 1 millivolt across a circuit of input capacity $100 \mu \mu \mathrm{f}$, with a chamber voltage of 500 volts, is $0.2 \times 10^{-15}$ farad. If the chamber had a total capacity of $200 \mu \mu$, this change represents one part in one million. Although a step change in capacity is an unlikely effect in a well-constructed chamber, the example serves to illustrate the mechanical stability requirements placed upon the chamber design, and indicates the possibility of extraneous signals being caused by mechanical shocks to the chamber.

\section{MAGNETIC INDUCTION}

As stated before, magnetic induction seems to be one of the most troublesome of all of the effects mentioned so far. In order to find the so-. lution, one needs to understand the problem.

The term magnetic induction is somewhat misleading, since it implies the absence of an electric field. Any time-varying magnetic field must co-exist with a time-varying electric field; the relations between the two are furnished by Maxwell's equations. The reason that this effect has been termed magnetic induction is probably because the approach to analyzing the problem becomes easier when one considers the magnetic field to be the cause, with voltages induced in conductors as the effect. There is no need to solve for the values of the electric fields surrounding the conductors.

Imagine a space in which there exists a time-varying magnetic field in the $z$-direction only. Also imagine a closed path in the $x-y$ plane, which may be circular or otherwise. One of Maxwell's equations in integral form states that the integral of the electric field around a closed path equals the negative time rate of change of the total enclosed magnetic flux

$$
\oint \overline{\mathrm{E}} \cdot \overline{\mathrm{d} l}=-\frac{\mathrm{d} \phi}{\mathrm{d} t}
$$

If this path is free space, an electric field exists such that its integral equals $-\frac{\mathrm{d} \phi}{\mathrm{dt}}$. If we now place a conductor, such as a piece of wire; along this path, but leave a small gap at one point, we may write

$$
\oint \overline{\mathrm{E}} \cdot \overline{\mathrm{d} l}=\int_{\text {wire }} \overline{\mathrm{E}} \cdot \overline{\mathrm{d} l}+\int_{\text {gap }} \overline{\mathrm{E}} \cdot \overline{\mathrm{d} l}=-\frac{\mathrm{d} \phi}{\mathrm{d} t},
$$


where the first term represents the integral taken along the path through the interior of the wire, and the second the integral taken across the gap. If the dimensions of the chosen path are small compared with a wavelength of any frequencies involved in the time variation of the fields, it may be as sumed that the presence of the gap will prevent any current from flowing in the wire. This would not be true at frequencies sufficiently high to permit the existence of standing waves along the wire. If standing waves do not exist, then the gap guarantees that the current density $\bar{i}$ in the conductor is zero. It follows from Ohm's law, $\vec{i}=\sigma \overline{\mathrm{E}}$, where $\sigma$ is the conductivity, that $\bar{E}$ must also be zero within the conductor. Therefore, the integral of $\bar{E}$ through the wire is zero, and

$$
\int \bar{E} \cdot \overline{d \ell}=V_{g a p}=-\frac{d \phi}{d t} .
$$

This is the voltage one would measure across the gap with a high impedance device, and is the familiar one for magnetically induced voltage. The important thing to note is that no current flows, and therefore the total magnetic flux linking the loop is unchanged by the presence of the loop.

If the gap is now closed, letting the conductor form a closed loop, current will flow in the loop, resulting in the creation of some magnetic flux which will partially cancel the incident flux. The total flux $\phi_{t}$ linking the loop will consist of two components, $\phi_{i}$ due to the currents in the distant source, and $\phi_{r}$ due to the current in the loop:

$$
\phi_{\mathrm{t}}=\phi_{\mathrm{i}}+\phi_{\mathrm{r}}
$$

It is assumed that the source of the original electromagnetic field is sufficiently far removed as to be unaffected by the closing of the loop, which is a very good assumption in the practical case. By the definition of inductance, $\phi_{r}=$ LI. If Ohm's law is multiplied on both sides by A,

$$
A \bar{i}=\sigma A \bar{E}=\bar{I}
$$

where I is the total current. At low frequencies, where the current density is uniform throughout the cross-sectional area of the wire, $A$ is the actual area. At high frequencies, where skin effect results in high current density near the surface, and nearly zero density at the center, A becomes the effective area, some value less than the actual area. If we integrate both sides around the wire

$$
\oint \bar{I} \cdot \overline{d l}=A \sigma \oint \bar{E} \cdot \overline{d l}=l I
$$

or

$$
I=\frac{A \sigma}{l} \oint \bar{E} \cdot \overline{d l}=\frac{1}{R}\left(-\frac{d \phi t}{d t}\right)
$$


since $R=\frac{l}{A \sigma}$ is the AC resistance of the loop. Substitution back in equa tion (2) gives

$$
\phi_{\mathrm{t}}=\phi_{\mathrm{i}}-\frac{\mathrm{L}}{\mathrm{R}} \frac{\mathrm{d} \phi_{\mathrm{t}}}{\mathrm{dt}}
$$

To simplify, let the time variation of all fields be sinusoidal; if $\phi_{t}=\phi_{t o} e^{\text {jot }}$, then we have $\frac{\mathrm{d} \phi_{\mathrm{t}}}{\mathrm{dt}}=\mathrm{j} \omega \phi_{\mathrm{t}}$, and

$$
\phi_{t}=\frac{\phi_{i}}{1+j \omega L / R}=\frac{\phi_{i}}{1+j Q}
$$

The process of closing the loop results in reducing the total magnetic flux $\phi_{t}$ by the magnitude of $1+j \Omega$.

If a piece of coaxial cable is formed into a loop with the ends brought near each other but not touching, there is no closed conducting path, and the total magnetic flux linking the loop will be only that due to a distant source. The voltage $V=j \omega \phi$ will appear across the ends of the shield, as well as across the ends of the center conductor. If the two ends of the shield are brought into contact, a closed conducting path is formed, and the flux linking the cable loop will be reduced by the factor $1+j Q$. The voltage across the ends of the center conductor will therefore be reduced by the same amount. Similarly, if one were to observe the voltage across the open ends of the shield while shorting the ends of the center conductor, one would again observe a decrease, although the $1+j Q$ factor would probably be less for the center conductor than for the shield.

A typical plot of the magnitude of $1+j Q$ versus frequency is shown in Fig. 2. This is a calculated curve for a piece of RG54 cable formed into a circular loop about 55 inches in diameter. The calculations were made using the expression for inductance of a circular loop on page 52 of Terman's handbook, 1 and the graphs for the AC resistance of a tubular conductor on page 33. The DC resistance of the shield was measured, from which the $A C$ resistance for various frequencies were calculated. The curve indicates the possibility of reducing by a very large amount the total flux linking a cable loop, for high frequencies, by the simple process of guaranteeing that the shield of the cable forms a closed loop. This has been verified experimentally many times and is a commonly accepted practice.

For illustrative purposes, let us consider a system consisting of an ion chamber, high voltage supply, and electrometer, as shown in Fig. 3 . It is assumed that the connections between the units are by means of coaxial cables, the shields of which are connected to the chassis or cases of the three units. As shown by the dotted line, if the electrometer chassis is connected to the high-voltage chassis, the shields of the cables, together with this added connection, form a closed conductive path adjacent in space to

1. F. E.Terman, "Radio Engineer's Handbook, "McGraw-Hill, New York, 1943, First Edition. 
the path followed by the center conductor of the cables. Adding the connection between high-voltage supply and electrometer will reduce the magnitude of the total flux linking the loop by the $1+j Q$ factor. The flux which remains, $\phi_{t}$, will induce a voltage into the loop formed by the center condductors of the cables, and closed through the output impedance of the highvoltage supply, the impedance between the two chamber connections, and the input impedance of the electrometer. This induced voltage, proportional to jw $\phi_{t}$, will distribute itself across the three impedances mentioned, proportional to their value, as shown in the equivalent circuit of Fig. 4. Since the output impedance of a good high-voltage supply is low over a broad frequency band, and since the impedance of the chamber usually looks like pure capacity and is low at sufficiently high frequencies, there may be a range of frequencies for which most of the induced voltage will appear across the input of the electrometer. This serves to emphasize the need for minimizing $\phi_{t}$ in the first place, for the input voltage sensitivity of electrometers may easily be as $10 \mathrm{w}$ as 1 to 10 millivolts. For a period channel, the figure might be in the microvolt region.

In considering the reduction of the total resultant flux $\phi_{t}$ by reducing loop area, it may occur that, although $\phi_{i}$ is certainly reduced with a decrease in loop area, it is also true that the inductance of the loop, and therefore $Q$, will also be reduced. Thus one might expect to find either an optimum spacing for the conductors of the loop, or at least a spacing below which little advantage is gained. This does not appear to be the case, for a change in loop inductance and $Q$ is much less significant than the change in the linking incident flux $\phi_{i}$.

The system of Fig. 3, which provides for pickup reduction by the process of providing a closed shield path, will usually be found to be ineffective at low frequencies where the $1+j Q$ factor approaches unity. A system which serves to essentially eliminate the loop entirely over a much broader range of frequencies is shown in Fig. 5. The only difference from the system in Fig. 3 consists of the addition of the elements $R$ and $C$ within the ion chamber housing. If we now attempt to evaluate the integral of $E$ around the center conductor loop, starting, say, at the chassis of the highvoltage supply, following through $Z_{\text {out }}$, through the high-voltage cable center conductor, and through $R$, we arrive at the junction of $C$ and the chamber capacity $\mathrm{C}_{C}$. We are now presented with the choice of completing the loop in either of two ways: (1) through $C_{C}$, center conductor of signal cable, and $\mathrm{Z}_{\text {in }}$ or (2) through $\mathrm{C}$, shield of signal cable back to starting point. The choice of path will not affect the total value of the line integral, since both paths are nearly adjacent, but choosing the second path through $C$ reveals that practically all of the induced voltage will appear across $R$, providing only that it be large compared with the reactance of $C$ and $Z_{\text {out }}$. It is not necessary that it be large compared with $Z_{i n}$. This arrangement will provide pickup reduction down to the frequency where the reactance of $C$ equals $R$. 
The effectiveness at high frequencies depends upon keeping the reactance of the physical capacitor C low, which suggests using capacitors with high self-resonant frequencies, or parallel combinations of various capacitors with staggered self-resonant frequencies. The closing of the shield path a round the loop in Fig. 5, although shown, is not a necessity. It may serve as double protection.

\section{GROUND LOOPS}

A condition that may multiply magnetic induction problems, as well as introduce new ones, may axise from multiple ground connections in a system. In the system of Fig. 3 , it is probable that either the high-voltage unit, or the electrometer, or both, will be AC operated. If so, their chassis are provided with a path to ground through the stray capacity of the power transformer and the $A C$ power leads. If the chamber were connected to ground, an additional loop for magnetic induction would be created. This would be the path through the center conductor of the signal cable to the electrometer, through its input impedance to chassis, through power transformer capacity and AC power cord to ground, through ground back to chamber, and finally, through the stray capacity in the chamber to the signal cable. The ground path could result in the introduction of IR voltages into the loop, since ground may carry quite a variety of currents. The generally accepted procedure to avoid ground loops consists of guaranteeing only a single ground connection to the entire system. In this example, the logical place to ground the system would be at the location of the high voltage and electrometer units. There are several reasons for this. First, these are the units nearest to ground initially: second, it may be desirable to mount them in a rack cabinet, in which case grounding is required for the sake of safety. Third, the chamber, usually the smallest item, is the easiest to insulate from any connection to ground.

\section{CONCLUSION}

The various problems just discussed in connection with requirements for RF filtering, noise currents from cables, stability of ion chamber voltages, magnetic induction, and ground loops, have been found to be nearly the rule, not the exception, in reactor control instrumentation. In a very large percentage of the instances where large induction loops were formed in the layout of the system, or other improper procedures followed, trouble has resulted. In some instances the great difficulties involved in trying to re route cables in existing systems, or make other changes, has made it completely impractical to even attempt correction of the troubles. In such cases proper system layout would almost certainly have avoided the troubles easily. 
The magnetic induction and ground loop problems are likely to occur in almost any type of low-level detection system, whether the signal being detected be a pulse, sine wave, or small DC voltage or current, and certainly are not restricted to reactors, or radiation detection systems.

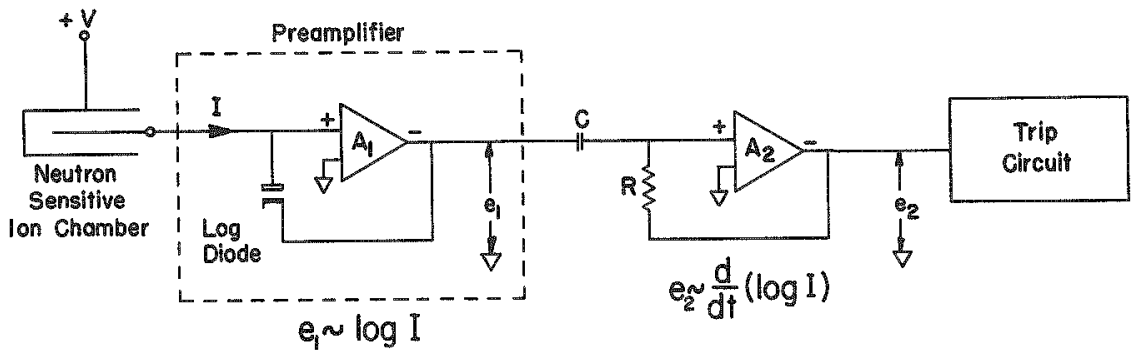

FIG. 1

BLOCK DIAGRAM OF PERIOD CHANNEL



FIG. 2

PLOT OF ATTENUATION FACTOR $11+i Q \mid$ VS. FREQUENCY FOR SHIELD BRAID OF 55 INCH DIAMETER LOOP OF RG-54

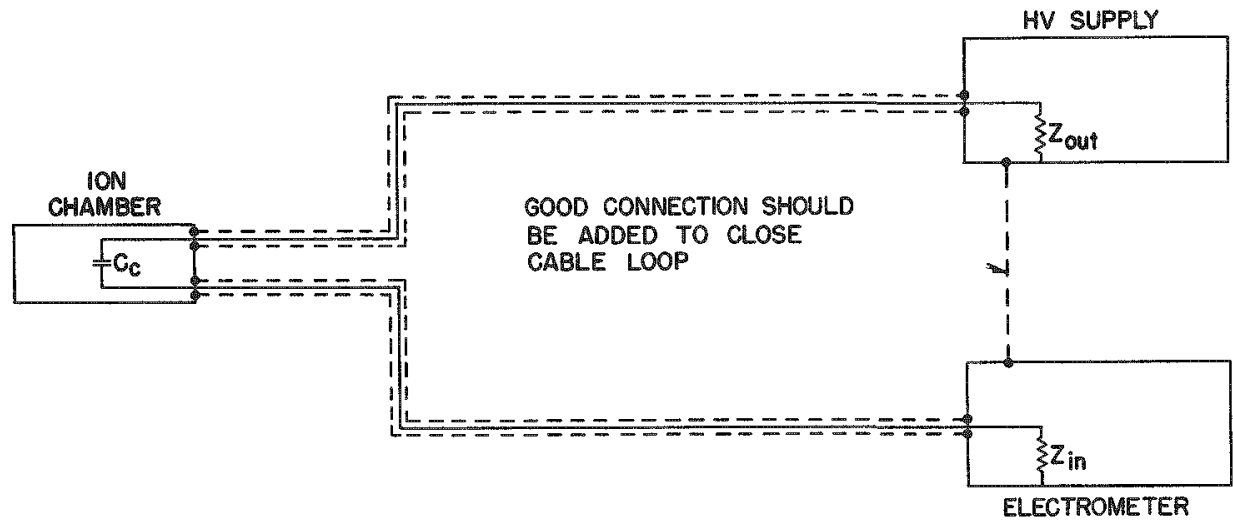

FIG. 3

REDUCTION OF PICKUP BY CLOSING SHIELD LOOP 


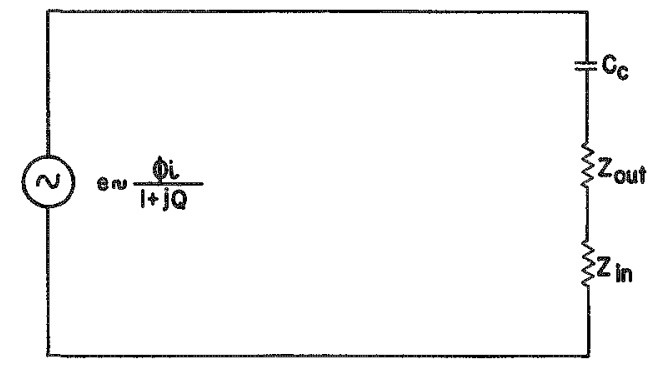

F10. 4

EQUIVALENT CIRCUIT AROUND CENTER CONDUCTOR

PATH OF FIG. 3



FIG. 5

REDUCTION OF PICKUP BY LOOP ISOLATION

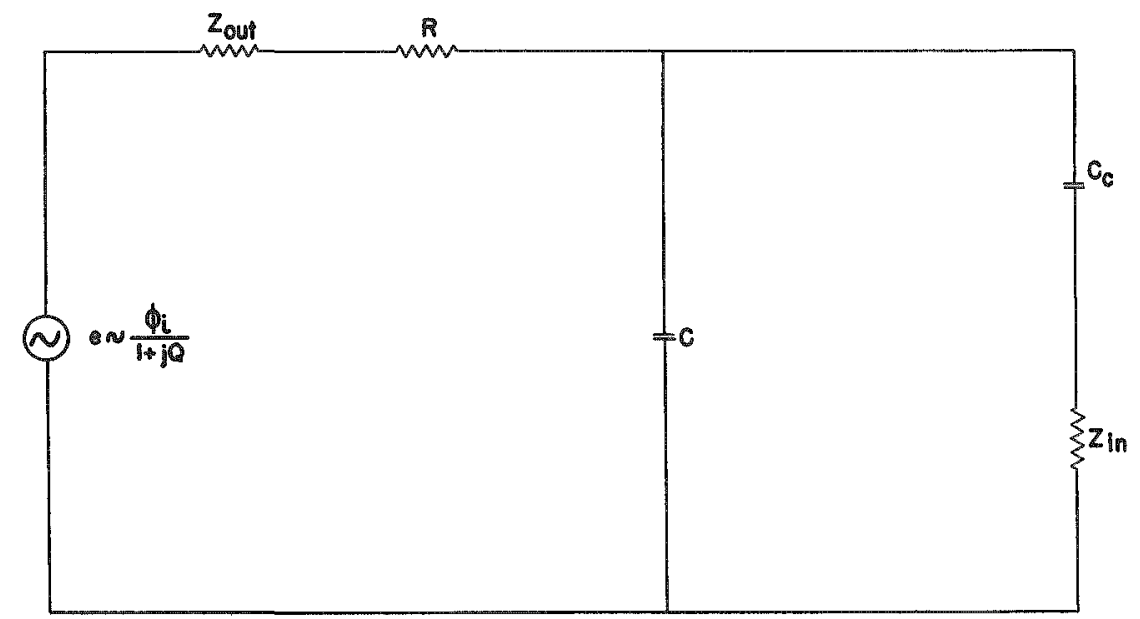

FIG. 6

APPROXIMATE EQUIVALENT CIRCUIT OF FIG. 5 